\title{
Nuevos pseudocóccidos (Hemiptera: Pseudococcidae) y sus hospedantes para la provincia de Sancti Spíritus, Cuba
}

\author{
New mealybugs (Hemiptera: Pseudococcidae) and host plants for Sancti Spíritus Province, Cuba \\ RAÚL MIRABAL-RODRÍGUEZ1, MARCOS TULIO GARCÍA-GONZÁLEZ², \\ LEÓNIDES CASTELLANOS-GONZÁLEZ ${ }^{3}$, YANDER FERNÁNDEZ-CANCIO ${ }^{4}$ y NANCY PÉREZ-REYES ${ }^{5}$
}

\begin{abstract}
Resumen: El presente estudio tuvo como objetivo informar sobre los nuevos pseudocóccidos y sus hospedantes para la provincia de Sancti Spíritus (Cuba) durante el período 2005-2015. En estos diez años se informaron 10 géneros y 21 especies de pseudocóccidos (16 nuevos informes) en los ecosistemas atendidos por la Estación Territorial de Protección de Plantas de Fomento, sobre 130 especies de plantas hospedantes, agrupadas en 57 familias botánicas. Se registra un incremento notable de los géneros y especies de pseudocóccidos presentes, así como de sus plantas hospedantes, muchas de las cuales no estaban informadas para Cuba.
\end{abstract}

Palabras clave: Entomología, insectos, cochinillas, diversidad.

\begin{abstract}
The aim of this paper is to provide information regarding a new registry of mealybugs and host plants in the Sancti Spíritus province of Cuba between 2005-2015. In this time period, 10 genera and 21 species of mealybugs were found (16 new records) in the ecosystems of the area studied by the Fomento Territorial Plant Protection Station. These new registries were found on 130 host plants species ( 40 previously unrecorded for Cuba), from 57 botanical families. This represents a notable increase in the number of genera and species of mealybugs present, as well as in the number of host plants, many of which were previously unreported in Cuba.
\end{abstract}

Key words: Entomology, insects, cochineals, diversity.

\section{Introducción}

Hemiptera: Pseudococcidae forma un grupo importante de insectos, conocidos como chinches harinosas o algodonosas, o simplemente cochinillas. Según Niebla et al. (2010), se conocen en el mundo un estimado total de 271 géneros y 2188 especies, de éstas varias tienen un marcado interés agrícola. Los primeros antecedentes de estudios taxonómicos de la familia Pseudococcidae en Cuba se remontan a inicios del siglo XX y corresponden a Ballou (1926) (Bruner et al. 1975), sin embargo Martínez et al. (2008), señalan que las colecciones cubanas atesoran tan solo una parte del material de referencia, ya que la mayor proporción de estos especímenes se encuentran depositados fundamentalmente en las colecciones de los Estados Unidos de Norte América.

Hace algunos años se publicó un libro sobre las chinches harinosas en Cuba que ha proporcionado claves (herramientas), que ayudan al diagnóstico certero y oportuno, producto de las observaciones de los ejemplares colectados en el país (Martínez 2012). Con este material se ha capacitado al personal de las Estaciones Territoriales de Protección de Plantas (ETPP) para la clasificación las especies de este grupo de insectos.

La primera intercepción oficial de una cochinilla harinosa en el territorio de la ETPP de Fomento se registró el 28 de febrero de 2002 cuando se informó a Nipaecoccus nipae (Maskell) afectando mandarina (Citrus reticulata Blanco) y cacao (Theobroma cacao L.) (LAPROSAV 2015). Por otra parte son escasos los informes de trabajos que se han estado realizando en los últimos años sobre este grupo de insectos, que ha incrementado sus poblaciones asociado al cambio climático (Vázquez 2011).

El presente estudio tuvo como objetivo informar sobre los nuevos pseudocóccidos y sus hospedantes para la provincia de Sancti Spíritus, Cuba, durante el período 2005-2015.

\section{Materiales y métodos}

El trabajo se realizó en el municipio Fomento, provincia Sancti Spiritus, Cuba, en el período 2005 - 2015. Los especialistas de esta entidad aplican un Programa de Defensa contra Maconellicoccus hirsutus (Green) (chinche harinosa rosada), desde 1997.

Los muestreos abarcaron los 12 cuadrantes cartográficos del municipio. Las observaciones se dirigieron a los tallos, hojas, flores, frutos y raíces en los hospedantes preferenciales de las cochinillas y en plantas de cultivo de interés económico para el territorio. El muestreo abarcó además la vegetación espontánea. La frecuencia del monitoreo obedeció al nivel de riesgo declarado para la chinche harinosa rosada de cada cuadrante cartográfico (Navarro 2003).

\footnotetext{
${ }^{1}$ Máster en Ciencias agrícolas, Estación Territorial de Sanidad Vegetal (ETPP), Fomento, Sancti Spíritus, Cuba. ${ }^{2}$ Doctor en Ciencias Agrícolas, Universidad de Sancti Spíritus José Martí Pérez, Facultad de Ciencias Agropecuarias. avenida de los Mártires \# 360, Sancti Spíritus, Cuba, marcostg@uniss. edu.cu. ${ }^{3}$ Doctor en Ciencias Agrícolas, Universidad de Pamplona, Facultad de Ciencias Agrarias, Campus Universitario, Km 1, Vía Bucaramanga, Norte de Santander, Colombia, lclcastell@gmail.com. ${ }^{4}$ Máster en Ciencias Agrícolas, Universidad de Sancti Spíritus José Martí Pérez, Facultad de Ciencias Agropecuarias, avenida de los Mártires \# 360, Cuba, yanderfc@uniss.edu.cu. ${ }^{5}$ Máster en Agricultura Sostenible, Estación Territorial de Sanidad Vegetal (ETPP), Especialista Principal, Fomento, Sancti Spíritus, Cuba. Autor para correspondencia: Leónides Castellanos-González, Doctor en Ciencias Agrícolas, Universidad de Pamplona, Facultad de Ciencias Agrarias, Campus Universitario, Km 1 vía Bucaramanga, Norte de Santander, Colombia, lclcastell@gmail.com.
} 
En las plantaciones de cultivos económicos se empleó el método de diagonales dobles observando 25 plantas por ha y cinco plantas más por cada hectárea adicional. En plantas aisladas se procedió según la sintomatología o evidencia física de la presencia de insectos característicos de la familia.

Las muestras se conservaron en alcohol al $70 \%$, se etiquetaron según Modelo 10-0, asentándose en el Registro "Libro de envío de muestras de la Estación Territorial de Protección de Plantas (ETPP)" y fueron remitidas al Laboratorio Provincial de Sanidad Vegetal (LAPROSAV) de Sancti Spíritus según la Norma Cubana 486: 09 (MINAG 2009).

En el laboratorio, con auxilio de un pincel fino y aguja enmangada se separaron los individuos presentes en el material vegetal y se conservaron con sus respectivos datos.

Los especímenes adultos se trasladaron a tubos viales con una solución de cloral-fenol para el aclarado del cuerpo y la eliminación de suciedades. Los viales fueron colocados en baño María durante $10 \mathrm{~min}$ para acelerar el proceso. Luego se procedió a la limpieza de los insectos colocándolos en porta objetos excavados con una gota de lacto-fenol. Se les realizó una pequeña incisión entre el tercer par de patas y la región anal y se presionó suavemente para extraer el contenido de su interior. Una vez terminado este procedimiento se depositó la muestra en una gota de fucsina para su teñido y posterior identificación.

La clasificación taxonómica se realizó con la ayuda de las claves de Williams y Granara de Willink (1992) para Centro y Sudamérica, y los aportes sobre la identificación de pseudocóccidos en Cuba de Blanco et al. (2002) y Martínez (2012).
Para determinar la flora hospedera de pseudocóccidos, durante la prospección de los mismos, se puso especial interés a la individualización de la especie hospedante, para lo cual cuando necesario se tomaron partes de la planta que fueron depositadas en bolsas de nailon infladas y enviadas a LAPROSAV de Sancti Spíritus para su identificación hasta especie.

\section{Resultados y discusión}

El número de géneros y pseudocóccidos se ha incrementado en el tiempo en el territorio de la ETPP Fomento en un escenario donde existe una riqueza florística muy abundante, y que apunta hacia una agricultura más diversificada. Se identificaron 10 géneros y 21 especies de pseudocóccidos en los ecosistemas del territorio. En el período de 10 años se interceptaron 16 especies pertenecientes a ocho géneros que constituyen nuevos informes para el municipio, lo que fue corroborado con el Registro Territorial de Plagas de la ETPP Fomento y también por el LAPROSAV (2015) para la provincia de Sancti Spiritus (Tabla 1).

En el período evaluado se informaron 130 especies de plantas hospedantes de pseudocóccidos agrupadas en $57 \mathrm{fa}-$ milias botánicas. Estos resultados avalan lo planteado por Niebla et al. (2010), al referir un alto potencial de hospedantes de esta familia de insectos para el archipiélago cubano. Se verificó, asimismo, que 44 de los 130 hospedantes informados para el territorio del Municipio Fomento (Tabla 2), no aparecen referidos en los estudios realizados por Blanco et al. (2002), donde se informan 16 especies de pseudocóccidos y

Tabla 1. Especies de pseudocóccidos (Hemiptera: Pseudococcidae) registradas en el periodo 2005-2015 en el territorio de la ETPP, Fomento, Sancti Spíritus, Cuba.

\begin{tabular}{|c|c|}
\hline Género & Especie \\
\hline Nipaecoccus Sulc, 1945 & Nipaecoccus nipae (Maskell) \\
\hline \multirow[t]{4}{*}{ Dysmicoccus Ferris, 1950} & Dysmicoccus brevipes (Cockerell) \\
\hline & Dysmicoccus alazon Williams \\
\hline & Dysmicoccus bispinosus (Beardsley)* \\
\hline & Dysmicoccus sp.* \\
\hline \multirow[t]{2}{*}{ Pseudococcus Westwood, 1840} & Pseudococcus elisae Borchsenius* \\
\hline & Pseudococcus longispinus (Targioni) \\
\hline Kiritshenkella Borchsenius, 1948 & Kiritshenkella sacchari (Green)* \\
\hline Saccharicoccus Ferris, 1950 & Saccharicoccus sacchari (Cockerell) \\
\hline \multirow[t]{2}{*}{ Paracoccus Ezzat \& McConell, 1956} & Paracoccus marginatus Williams y Granara de Willink* \\
\hline & Paracoccus sp.* \\
\hline \multirow[t]{4}{*}{ Phenacoccus Cockerel, 1893} & Phenacoccus solani Ferris* \\
\hline & Phenacoccus solenopsis (Tinsley)* \\
\hline & Phenacoccus madeirensis (Green)* \\
\hline & Phenacoccus sp.* \\
\hline \multirow[t]{3}{*}{ Ferrisia Fullaway, 1923} & Ferrisia virgata (Cockerell)* \\
\hline & Ferrisia consobrina (Williams y Watson)* \\
\hline & Ferrisia sp.* \\
\hline \multirow[t]{2}{*}{ Planococcus Ferris, 1950} & Planococcus minor (Maskell)* \\
\hline & Planococcus citri (Risso)* \\
\hline Maconellicoccus Ezzat, 1958 & Maconellicoccus hirsutus (Green)* \\
\hline
\end{tabular}

* Nuevos informes en el período 2005-2015. 
Tabla 2. Especies de pseudocóccidos (Hemiptera: Pseudococcidae) por plantas hospedantes en el territorio de la ETPP, Fomento, Sancti Spíritus, Cuba.

\begin{tabular}{|c|c|c|c|}
\hline \multirow{2}{*}{ No } & \multicolumn{2}{|r|}{ Hospedantes } & \multirow{2}{*}{ Especies de pseudocóccidos } \\
\hline & Nombre vulgar & Nombre científico & \\
\hline 1 & Name & Dioscorea alata L. & Dysmicoccus bispinosus / Dysmicoccus brevipes \\
\hline 2 & Achicoria & Cichorium intybus L. & Dysmicoccus alazon \\
\hline 3 & Culantro & Eryngium foetidum $\mathrm{L}$. & Planococcus citri \\
\hline 4 & Esclaviosa & Capraria biflora $\mathrm{L}$. & Phenacoccus madeirensis \\
\hline 5 & Llantén & Plantago major L. & Dysmicoccus bispinosus \\
\hline 6 & Manzanilla & Matricaria chamonilla $\mathrm{L}$. & Phenacoccus madeirensis \\
\hline 7 & Rompe zaragüey & Chromolaena odorata (L.) K. et Rob.. & Dysmicoccus alazon \\
\hline 8 & Albizia & Albizia lebbeck (L.) Benth & Dysmicoccus alazon / Nipaecoccus nipae \\
\hline 9 & Almendro & Termina liacatappa $\mathrm{L}$. & Dysmicoccus bispinosus / Dysmicoccus alazon / Phenacoccus solani \\
\hline 10 & Casco de buey & Bahuinia divaricata $\mathrm{L}$. & Dysmicoccus alazon / Nipaecoccus nipae \\
\hline 11 & Chote & Parmentiera edulis DC & Dysmicoccus bispinosus / Dysmicoccus alazon \\
\hline 12 & Copey & Clusia rosea Jacq. & Nipaecoccus nipae \\
\hline 13 & Eucaliptus & Eucaliptus sp. & Pseudococcus longispinus \\
\hline 14 & Guarana & Cupania americana $\mathrm{L}$. & Nipaecoccus nipae / Phenacoccus solenopsis \\
\hline 15 & Guásima varía & Luehea candida (M \& Ses ex DC) Mart. & Nipaecoccus nipae \\
\hline 16 & Sarzafrás & Burcera graveolens (H.B.K.) T \& P. & Dysmicoccus alazon \\
\hline 17 & Siguaraya & Trichilla habanensis Jacq. & Phenacoccus solenopsis \\
\hline 18 & Yamagua & Guarea guidonia Jacq. & Nipaecoccus nipae \\
\hline 19 & Bledo & Amaranthus dubius Mart. & Ferrisia virgata \\
\hline 20 & Canutillo blanco & Commelina diffusa Burn. & Dysmicoccus alazon / Phenacoccus sp. \\
\hline 21 & Amor seco & Desmodium canum J. 1. Sching & Phenacoccus solenopsis \\
\hline 22 & Maribari & Macroptyilium lathiroides (L) DC. & Dysmicoccus bispinosus \\
\hline 23 & Mastuerzo & Lepidium virginicum $\mathrm{L}$. & Dysmicoccus bispinosus \\
\hline 24 & Oro azul & P. nodiflora (L.) Greene & Dysmicoccus alazon \\
\hline 25 & Tábano & Pavonia fruticosa (Mill.) F. \&Rend. & Phenacoccus madeirensis \\
\hline 26 & Carambola & Averrhoa carambola L. & Planococcus minor / Pseudococcus longispinus \\
\hline 27 & Fresa & Fragaria vesca $\mathrm{L}$. & Dysmicoccus bispinosus \\
\hline 28 & Granada & Punica granatum $\mathrm{L}$. & Dysmicoccus brevipes / Dysmicoccus alazon \\
\hline 29 & Pomarrosa de Málaga & Eugenia malaccensis L. & Dysmicoccus alazon / Nipaecoccus nipae \\
\hline 30 & Aglonema & Aglaonema sp. & Nipaecoccus nipae \\
\hline 31 & Aralia & Polyscias variegatum L. & Nipaecoccus nipae \\
\hline 32 & Aralia lea & Leea rubra $\mathrm{L}$. & Dysmicoccus brevipes / Dysmicoccus alazon \\
\hline 33 & Cordón de seda & Argyreia nervosa (Burm. f) Boj. & Dysmicoccus alazon \\
\hline 34 & Erantemo & Pseudoeranthemum bicolor Radk & Nipaecoccus nipae \\
\hline 35 & Espuelita & Delphinium consolida L. & Dysmicoccus bispinosus \\
\hline 36 & Heliconia & Heliconia $\mathrm{sp}$. & Dysmicoccus sp. \\
\hline 37 & Maena lila & Thumbergia erecta T. Anders & Ph. madeirensis / Dysmicoccus alazon \\
\hline 38 & Malanguita & Syngonium podophyllum Schott & Nipaecoccus nipae / Pseudococcus longispinus \\
\hline 39 & Palma bambú & Chamaedorea seifritzii $\mathrm{L}$. & Dysmicoccus brevipes / Dysmicoccus alazon \\
\hline 40 & Rabo de gato & Achyranthes aspera var. indica $\mathrm{L}$. & Dysmicoccus brevipes / Phenacoccus solenopsis / Ferrisia virgata \\
\hline 41 & Siempre viva & Helychrysum bracteatum (Vent.) Andr. & Dysmicoccus alazon \\
\hline 42 & Bejuco guajaca & Tillandsiaus neoides L. & Dysmicoccus alazon \\
\hline 43 & Bejuco baracoa & Rourea glabra Kunth. & Nipaecoccus nipae \\
\hline 44 & Tabaco & Nicotiana tabacum L. & Planococcus citri \\
\hline
\end{tabular}

69 hospedantes, y Mestre et al. (2010), con 173 especies de 14 familias. Estos trabajos recogen los estudios más comple- tos que anteceden estos resultados para pseudocóccidos en Cuba. 
Por su parte Vázquez (2011) señala que las principales evidencias en Cuba sobre dispersión de plagas de insectos exóticos tiene una alta relación con los eventos extremos del cambio climático y pone ejemplo de especies de pseudocóccidos que han incrementado sus poblaciones en el país tanto después de eventos extremos como los huracanes, que han causado la disminución de sus biorreguladores naturales, como después de intensas sequías, que favorecieron el incremento de sus poblaciones.

Nuestros resultados ponen de evidencia el nivel de incremento de los pseudocóccidos en los últimos años en un territorio bastante pequeño de la isla de Cuba y constituye una alerta para el país y la región centroamericana y del Caribe por las posibilidades de dispersión tan alta de estos insectos y la ampliación del rango de sus plantas hospedantes.

\section{Conclusiones}

En el período de diez años se ha producido un incremento notable de los géneros y especies de hospedantes de pseudocóccidos en los ecosistemas del territorio de la ETPP Fomento, así como de sus plantas hospedantes, muchas de las cuales no estaban informadas anteriormente para Cuba.

\section{Literatura citada}

BALLOU, C. H. 1926. Los cóccidos de Cuba y sus plantas hospederas. Boletín Estación Experimental Agronómica de Santiago de Las Vegas 51: 1-47.

BLANCO, E. R.; PÉREZ, I.; RODRÍGUEZ, A. 2002. Encuesta de los pseudocóccidos de Cuba. Resultados del período 20012002. Fitosanidad 7 (2): 37- 40.

BRUNER, S. C.; SCARAMUZZA, L. C.; OTERO, L. C. 1975. Catálogo de insectos que atacan a las plantas económicas de Cuba, 2 da Ed. revisada y aumentada, Instituto de Zoología, Academia de Ciencias de Cuba, La Habana. 395 p.
LAPROSAV. 2015. Laboratorio Provincial de Sancti Spiritus. Registro Territorial Histórico de Plagas. Departamento Fitosanitario Provincia Sancti Spiritus. Cuba. 25 p.

MARTÍNEZ, M. A.; BLANCO, E.; SURÍS, M. 2008. Fauna de chinches harinosas (Hemiptera: Pseudococcidae) asociadas a las plantas de interés: IV Plantas ornamentales. Protección Vegetal 23 (1): 48-53.

MARTÍNEZ, M. A. 2012. Las chinches harinosas en Cuba. Tesis en opción al título de doctor en Ciencias. Protección Vegetal 27 (1): 65.

MESTRE, N.; HODGES, G. S.; VEITÍA, M.; CERNUDA, P.; HERRERA, P. 2010. Nuevos registros de insectos escamas (Hemiptera). Fitosanidad 14 (3): 181-183.

MINAG. 2009. Norma Cubana 486. Ministerio de la Agricultura Cuarentena Vegetal - Embalaje y traslado de productos básicos para análisis de laboratorio - Reglas Generales. La Habana, 14 p.

NAVARRO, A. 2003. Evaluación y categorización del riesgo fitosanitario territorial para la vigilancia contra la cochinilla rosada (Maconellicoccus hirsutus (Green)) en Cuba. CNSV. 35 p.

NIEBLA, S.; JIMÉNEZ, J.; CASTELLANOS, L.; SUÁREZ, E. 2010. Pseudocóccidos en la provincia de Cienfuegos y sus hospedantes. Fitosanidad 14 (1): 3-9.

VÁZQUEZ, L. L. 2011. Cambio climático, incidencia de plagas y prácticas agroecológicas resilientes. Instituto de Investigaciones de Sanidad Vegetal, La Habana. 242 p.

WILLIAMS, D. J.; GRANARA DE WILLINK, M. C. 1992. Mealybugs of Central and South Americ, CAB International.

Recibido: 27-ago-2017 • Aceptado: 29-jun-2018

Citación sugerida:

MIRABAL-RODRÍGUEZ，R.; GARCÍA-GONZÁLEZ， M. T.; CASTELLANOS-GONZÁLEZ, L.; FERNÁNDEZ-CANCIO, Y.; PÉREZ-REYES, N. 2018. Nuevos pseudocóccidos (Hemiptera: Pseudococcidae) y sus hospedantes para la provincia de Sancti Spíritus, Cuba. Revista Colombiana de Entomología 44 (2): 193-196. Julio-Diciembre 2018. 